\title{
Judged Consonance of Tactile and Auditory Frequencies
}

\author{
Ryuta Okazaki ${ }^{1}$, Taku Hachisu ${ }^{1,2}$, Michi Sato ${ }^{1,2}$, Shogo Fukushima ${ }^{1,2}$, \\ Vincent Hayward ${ }^{3}$, Hiroyuki Kajimoto ${ }^{1,4}$ \\ ${ }^{1}$ The University of Electro-Communications, ${ }^{2}$ Japan Society for the Promotion of Science, \\ ${ }^{3}$ UPMC Univ. Paris 6, Institut des Systemes Intelligents et de Robotique, ${ }^{4}$ Japan Science and Technology Agency
}

\begin{abstract}
With the aim of augmenting auditory sensation by tactile stimuli, we investigated cross-modal relationships between the two modalities, focusing on frequency. The results showed that frequency consonance between tactile and audio stimuli depends on the relationship between harmonics, in a manner similar to auditory waves, but with broader peaks.
\end{abstract}

KEYWORDS: cross-modal interaction, frequency perception, tactile-audio interaction,

INDEX TeRMS: H.5.2 [Information Interfaces And Presentation]: User Interfaces-Auditory (non-speech) feedback; H.5.2 [Information Interfaces And Presentation]: User InterfacesHaptic I/O

\section{INTRODUCTION}

Today, mobile devices come embedded with high-definition visual displays such as the "retina" display in iPhones (Apple Inc.). In contrast, high-definition sound is not always available in mobile devices. For example, low frequency components are absent because a bass speaker required for low frequency generation occupies a large space and activities such as walking may not be suited to wearing headphones. Low frequency components moreover can be hard to hear in noisy environments. These circumstances suggest the need to enhance audio on mobile devices in a different way.

The purpose of this research is to augment auditory sensation by vibrotactile stimuli and ultimately achieve "not physically audible but subjectively hearable tactile" vibration. The tactileauditory conversion had been proposed for the hearing-impaired [1][2], but our purpose is to develop this cross-modal interaction between tactile and auditory sensing for use by the general public. Although constrained in size, mobile devices have certain advantages in tactile stimulation because these are always held when in use.

There are several studies suggesting tactile-audio cross-modal interactions. Suzuki et al. reported that tactile roughness perception is modified by adding task-irrelevant sound [3]. Yau et al. clarified that the subjective auditory intensity is affected by simultaneously presenting tactile stimuli [4]. Each factor of the tactile-audio interaction such as phase, synchrony, and frequency were also studied [5][6][7][8]. Physiological studies reported that

1-5-1 Chofugaoka, Chofu, Tokyo 182-8585, Japan

The University of Electro-Communications

E-mail: okazaki@kaji-lab.jp tactile and auditory sensations share a common neural mechanism [9].

In our previous paper, we reported that the subjective auditory intensity, which is one of the basic perceptual attributes, becomes louder by adding tactile vibration using the same source as the sound [10].

However, for tactile stimuli, many investigations used white noise or signals using the same source as sound, as their primary interest was eliciting a subjective loudness. In contrast, another important attribute, frequency, is commonly perceivable by auditory and tactile modality. Naturally, the question arises whether we find a tactile-auditory relationship in the frequency region. This paper tries to answer this question.

\section{EXPERIMENT 1}

In the first experiment, we investigated whether the notion of "same frequency" exists between tactile and audio perception. Participants were asked to adjust the frequency of a tactile stimulus to reproduce the same feeling as with an accompanying auditory stimulus.

\subsection{Experimental Conditions}

Apparatus: The setup comprised a computer with two audio channels running Pure Data freeware (http://puredata.info/) to synthesize the stimuli. One audio channel powered the two sides of a set of high-quality headphones (QuietComfort, Bose Inc., USA), with strong active noise cancellation; the other channel was connected to an audio amplifier (RSDA202, Rasteme Systems Inc., Japan) driving a vibrotactile transducer (Haptuator mark2, Tactile Labs, Canada).

Participants: 28 participants, aged between 17 and 32 years. Each reported no auditory or tactile impairment.

Stimuli: To explore the frequency relationship between tactile and auditory sensation, four sinusoidal pure tones (100, 200, 400, and $800 \mathrm{~Hz}$ ) were used as standard auditory stimuli. The signal intensity was set to about $55 \mathrm{~dB}$ spl. For tactile stimulus comparison, we generated sinusoidal vibrations that were frequency-adjustable by the participants over the range 1 to 800 $\mathrm{Hz}$. The signal magnitude was set to $2 \mathrm{~m} / \mathrm{s}^{2}$ at $200 \mathrm{~Hz}$.

Procedure: While seated, each participant wore headphones and held the actuator with their left hand. One of the four standard auditory stimuli and a randomly selected initial tactile stimulus (50, 250, and $450 \mathrm{~Hz}$ ) were presented simultaneously and continuously. By using a graphic user interface, each was instructed to adjust the vibration frequency until the tactile vibration felt the same as the audio stimulus. No adjustment time limit was set, but the task was completed in less than 30 seconds. To measure a naive frequency relationship between tactile and auditory sensation, each participant was given a single stimulus. We did not give further stimuli to avoid the possibility that participants would subjectively employ an ordinal scale, which would not have been in accord with the purpose of the experiment, 
i.e. to see if there is a common absolute scale between the two modalities.

\subsection{Result and Discussion}

The participant responses from experiment 1 are shown in Figure 1. The vertical axis indicates the frequency of the stimulus and horizontal axis is the participant's number. The blue dots mark the frequency of the standard auditory stimulus given and the red bars represent the frequency of the tactile stimulus that the participant selected.

The experimental result showed that the frequency of the auditory stimulus and that of the tactile stimulus (participants' selection) were not well correlated. Even in the frequency range of $50-300 \mathrm{~Hz}$ that are differentiable tactile sensation [11], frequencies were not correlated well. From these findings, we infer that simple tactile-auditory frequency matching is not applicable.

Nevertheless, in the preliminary experiment after experiment 1 , some interesting comments were obtained that tactile and auditory frequencies felt the "same" when the frequency of the tactile stimulus was inter-related to that of the auditory stimulus, regardless of their actual frequencies (i.e. auditory: $100 \mathrm{~Hz}$, tactile: 50, 100, 200 or $300 \mathrm{~Hz}$ ). The result of experiment 1 also seems to support this comment, for example, participants 2 and 6 selected a $300 \mathrm{~Hz}$ tactile vibration in response to the $100 \mathrm{~Hz}$ auditory signal, whereas participants 9,22 , and 25 matched up a $100 \mathrm{~Hz}$ tactile vibration to the 200 and $800 \mathrm{~Hz}$ auditory signals, and participant 5 matched a $50 \mathrm{~Hz}$ tactile vibration to the $100 \mathrm{~Hz}$ auditory signal.

This suggests that there might be tactile-auditory frequency matching relation that depends not on their actual pitch but rather on whether these are harmonically related.

It is known that every sound in nature contains harmonic structure, a consequence of physical vibrational resonances. We encounter these harmonic structures "naturally" in daily life, and for the auditory system leads to the notion of consonance [12]. As we touch everyday objects that vibrate and produce sound at the same time, we might be extending this harmonic perception to tactile vibration and auditory signals. Based on these considerations, we proceed to the next experiment applying the notion of consonance.

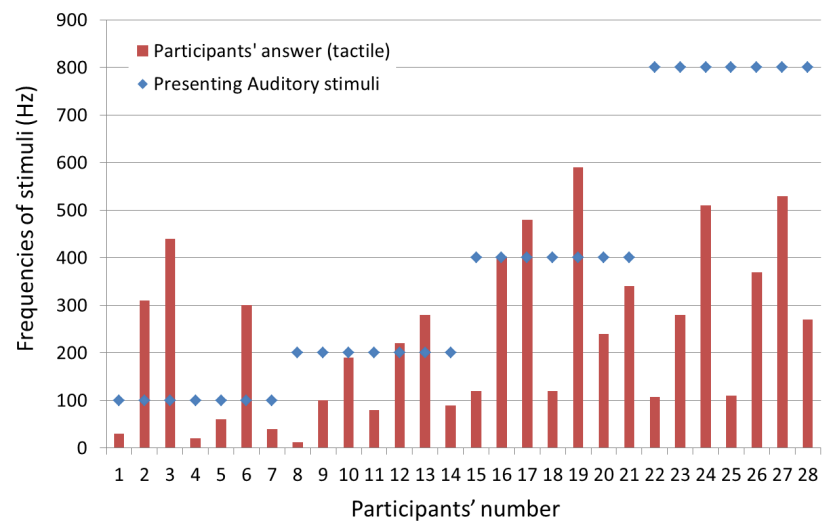

Figure 1. Result of experiment 1. Participants' responses were uncorrelated with actual frequencies of standard auditory stimuli.

\section{EXPERIMENT 2}

Two sub-experiments were carried out using the notion of consonance. In experiment $2 \mathrm{~A}$, perceived consonance between tactile and auditory stimuli was measured. To compare the result with ordinary auditory consonance, experiment $2 \mathrm{~B}$ was also conducted with the same procedure but using only auditory stimuli.

\subsection{Experimental Conditions}

Apparatus: The same setup as in experiment 1 was employed, with exception that in experiment $2 \mathrm{~B}$ a pair of auditory stimuli were only via the headphones.

Participants: 9 volunteers, aged between 22 and 28 years, conducted experiment $2 \mathrm{~A}$ and $2 \mathrm{~B}$. None reported auditory or tactile impairments.

Stimuli: Saw-tooth sound waves with base frequencies 58.2, $65.4,73.4,82.4,92.4,103.5,116.5,130.8,146.8,164.8,184.9$, 207.6, 233.0, 261.6, 293.6 Hz were used as auditory stimuli, and saw-tooth vibrational waves with a base frequency $130.8 \mathrm{~Hz}$ was employed as a tactile stimulus. With these frequencies chord structures with a central frequency of $130.8 \mathrm{~Hz}$ could be formed. Pairing 65.4 and $130.8 \mathrm{~Hz}, 130.8$ and $130.8 \mathrm{~Hz}, 261.6$ and 130.8 $\mathrm{Hz}$ are perfect prime or perfect octaves with respect to the base harmonics. Others were in imperfect consonance or in dissonance (see Table 1). The reason we used saw-tooth waves instead of sinusoidal pure tones was that saw-tooth waves contain higher harmonic waves producing a more natural sound so that participants could more easily sense consonance between tactile and auditory stimuli using their harmonic structure as sensory clues. Fifteen consonant, imperfect consonant, or inconsonant pairs of tactile and auditory stimuli were prepared. Our expectation was that, for example, the pairing of the $65.8 \mathrm{~Hz}$ auditory stimulus with the $130.8 \mathrm{~Hz}$ tactile stimulus would be sensed as consonant because these are in the same harmonic structure. The signal magnitudes were set to about $60 \mathrm{~dB}$ spl for the auditory stimulus and set to $2 \mathrm{~m} / \mathrm{s}^{2}$ for the tactile stimulus at central frequency $(130.8 \mathrm{~Hz})$.

In experiment $2 \mathrm{~B}$, the frequencies of stimuli were identical except that both were auditory stimuli.

Procedure: As before, participants were seated, and wore headphones and hand-held actuators. They were instructed to respond with consonance between sound and vibration. One of the auditory stimuli (58.2 to $293.6 \mathrm{~Hz})$ and tactile stimulus $(130.8 \mathrm{~Hz})$ were presented continuously. Participants judged their consonance and responded with an analogue scale. No time limit was set for the task, but all tasks were done within 30 seconds. All 15 pairs of stimuli were presented three times but randomly, 45 tasks in total.

In experiment $2 \mathrm{~B}$, they were instructed to respond whether two sounds were in consonance.

Table 1. Auditory stimuli. Consonance between $130.8 \mathrm{~Hz}$ tactile stimulus is represented by marks. $\circ$ :Perfect consonance, $\Delta$ :Imperfect consonance, $\times$ :Dissonance

\begin{tabular}{|c|c|c|c|c|c|c|c|c|}
\hline Stimuli No. & 1 & 2 & 3 & 4 & 5 & 6 & 7 & 8 \\
\hline Frequency (Hz) & 58.2 & 65.4 & 73.4 & 82.4 & 92.4 & 103.5 & 116.5 & 130.8 \\
Musical scale & A\# & C & D & E & F\# & G\# & A\# & C \\
Consonance & $\times$ & $\mathrm{O}$ & $\times$ & $\triangle$ & $\times$ & $\triangle$ & $\times$ & O \\
\hline Stimuli No. & 9 & 10 & 11 & 12 & 13 & 14 & 15 & \\
\hline Frequency (Hz) & 146.8 & 164.8 & 184.9 & 207.6 & 233.0 & 261.6 & 293.6 & \\
Musical scale & D & E & F\# & G\# & A\# & C & D & \\
Consonance & $\times$ & $\triangle$ & $\times$ & $\triangle$ & $\times$ & O & $\times$ & \\
\hline
\end{tabular}

\subsection{Results and Discussions}

To perform statistical analysis, the results of the analogue scale were normalized from 0.0 (minimum) to 10.0 (maximum). Figure 2 shows the average results among participants of experiment $2 \mathrm{~A}$ and $2 \mathrm{~B}$. The vertical axis represents consonance; the horizontal axis represents frequencies of the stimuli. The 
transition of consonance in experiment 2A (tactile-auditory) seems to be flat compared with that of $2 \mathrm{~B}$ (auditory-auditory). From solely this result, the harmonic structure of tactile and auditory stimuli does not seem to contribute to the evaluation of consonance.

However, from analyzing the results of experiment 2B, it was found that the participants can be classified into two groups, those who could judge consonance correctly when comparing pairs of imperfect consonance or dissonance, and those who could not. For example, participant 4 in Figure 3 responded that the pair of auditory stimuli at $82.4 \mathrm{~Hz}$ and $130.8 \mathrm{~Hz}$ had high consonance, which is correct. In contrast, participant 8 responded that the same stimuli had low consonance.

Based on these differences, all participants were divided into two groups; Group A (four participants with good auditory consonance perception) and Group B (five participants without good auditory consonance). Figure 4 and Figure 5 show the average results of each group for experiment $2 \mathrm{~A}$ and $2 \mathrm{~B}$.

We found from these figures that the difference in the two groups for experiment $2 \mathrm{~B}$ is reflected in the result of experiment 2A. For Group B, we could not find any relationship between the consonance responses of experiment $2 \mathrm{~A}$ and $2 \mathrm{~B}$. In contrast, for Group A, these consonance responses have an obvious correlation in the range $92.4 \mathrm{~Hz}$ to $184.9 \mathrm{~Hz}$. Figure 6 shows the correlation of Group A between auditory-tactile consonance (2A-A) and auditory-auditory consonance (2B-B) in the frequency region from $92.4 \mathrm{~Hz}$ to $184.9 \mathrm{~Hz}$. The Spearman rank correlation coefficient test indicates that this correlation was significant $\left(\mathrm{r}_{\mathrm{s}}=\right.$ $0.82, \mathrm{p}<0.05$ ). In contrast, Figure 7 shows the correlation of Group $\mathrm{B}$ between auditory-tactile consonance (2A-B) and auditoryauditory consonance (2B-B) over the same frequency range. The test found no significance $\left(r_{s}=0.21, p>0.05\right)$. Thus, we conclude that participants who better judge the degree of consonance in auditory stimuli can also better judge the degree of consonance between tactile and auditory stimuli.

Now, a new question arises; why can some participants judge imperfect consonance whereas the other participants could not? We interviewed all participants after the experiments and one compelling difference was found; every participant in Group A had learned to play a wind instrument such as flute, saxophone, or euphonium. Previous studies suggested that the tactile sensation is used as feedback in acoustical playing [13][14]. We suggest that participants who could better judge consonance in auditory stimuli had acquired that skill through practice playing a wind instrument, and possibly, also learned combinations of vibration and sound from their instrument, that may have contributed to their ability to judge consonance between tactile and auditory stimuli.

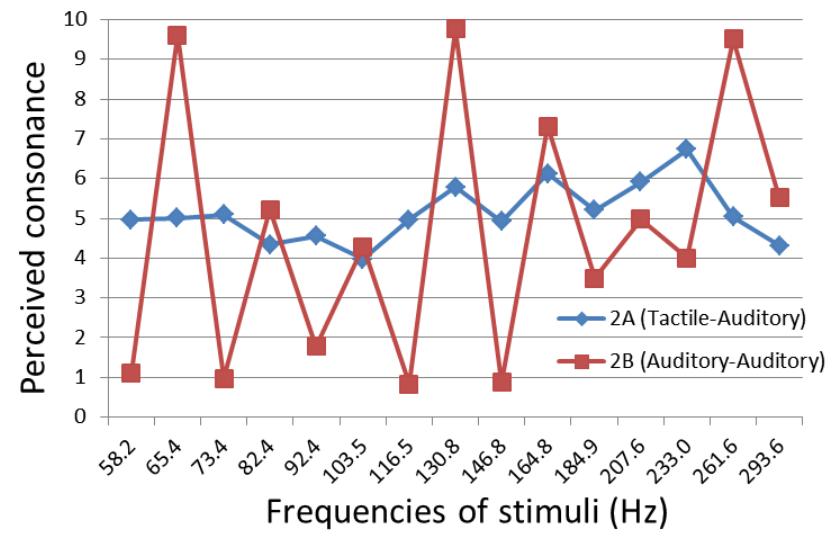

Figure 2. Average results of experiment $2 \mathrm{~A}$ and $2 \mathrm{~B}$.

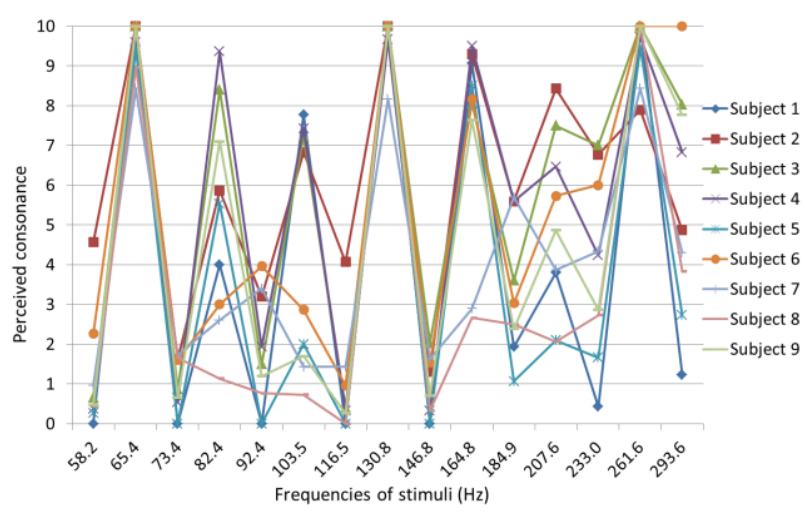

Figure 3 . Result of experiment $2 \mathrm{~B}$ by subject.

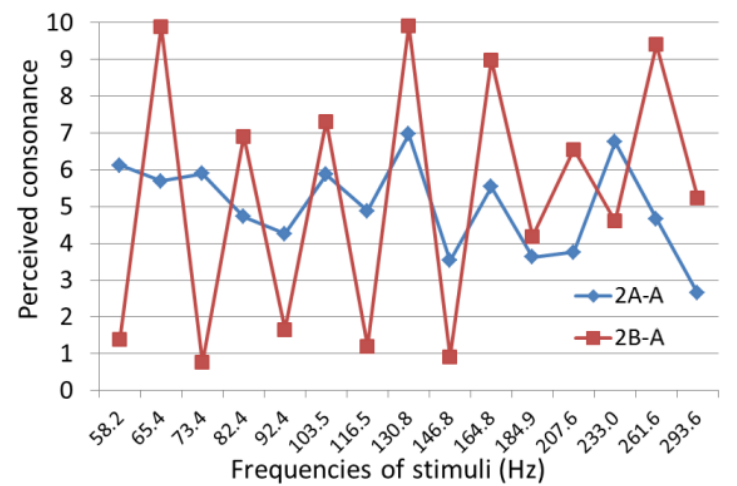

Figure 4. Average results of experiment 2 in Group A.

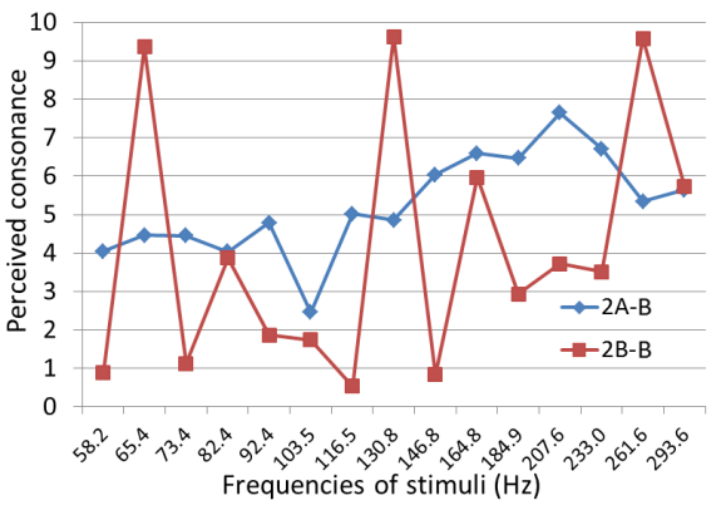

Figure 5. Average results of experiment 2 in Group B. 


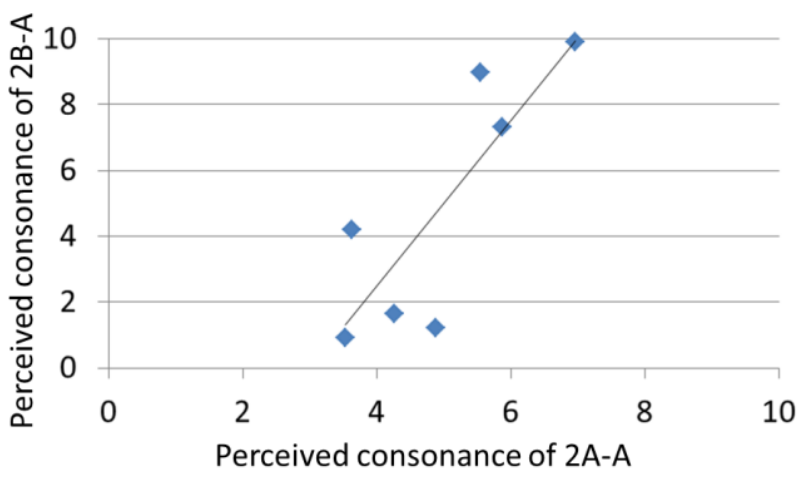

Figure 6. Correlation in perceived consonance between tactile-audio(2A-A) and audio-audio(2B-A) in frequency range from $92.4 \mathrm{~Hz}$ to $184.9 \mathrm{~Hz}$ (Group A)

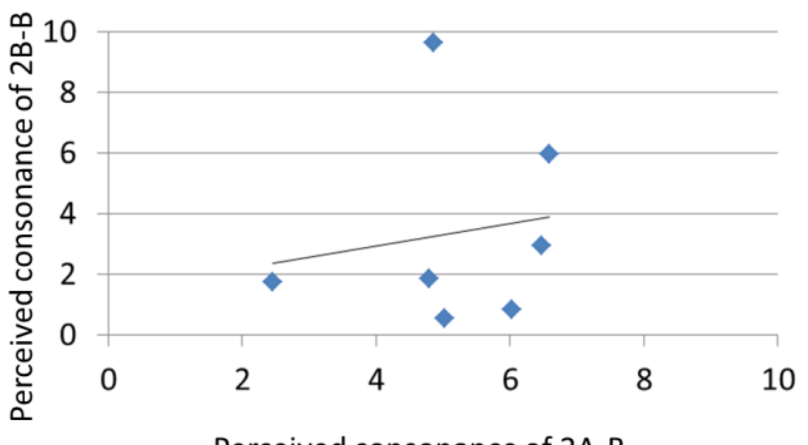

Perceived consonance of 2A-B

Figure 7. Correlation in perceived consonance between tactile-audio(2A-B) and audio-audio(2B-B) in frequency range from $92.4 \mathrm{~Hz}$ to $184.9 \mathrm{~Hz}$ (Group B)

\section{Conclusions}

In this paper, we investigated the possibility of tactile-audio crossmodal interaction in frequency perception. In exploring audio and tactile frequency relations, our results suggest that there might be a tactile-auditory frequency matching mechanism that is not dependent on actual pitch but rather depends on whether frequencies are in the same harmonic structure.

Based on this consideration, a further experiment was conducted from which the results showed that frequency consonance between tactile and auditory depended on harmonic content, rather than on actual frequency pitch. Also consonance acuity seems to require previous musical training experience.

\section{REFERENCES}

[1] O, Ozcan, E. Rebecca, and O. D. Kimbrough. Tactile Vocoders for the Deaf. Engineering in Medicine and Biology Magazine, IEEE, 6(3):37-42, September 1987.

[2] P. L. Brooks, B. J. Frost, J. L. Mason, and D. M. Gibson. Word and Feature Identification by Profoundly Deaf Teenagers Using the Queen's University Tactile Vocoder. Journal of Speech and Hearing Research, 30:137-141, March 1987.

[3] Y. Suzuki, J. Gyoba, and S. Sakamoto. Selective effects of auditory stimuli on tactile roughness perception. Brain Research, 1242:87-94, November 2008.

[4] J. M. Yau, A. I. Weber, and S. J. Bensmaia. Separate Mechanisms for Audio-Tactile Pitch and Loudness Interactions. Frontiers in Psychology, 1:160, October, 2010.
[5] J. M. Yau, J. B. Olenczak, J. F. Dammann, and S. J. Bensmaia1. Temporal frequency channels are linked across audition and touch. Current Biology, 19:561-566, April, 2009.

[6] E. C. Wilson, C. M. Reed, and L. D. Braida. Integration of auditory and vibrotactile stimuli: Effects of phase and stimulus-onset asynchrony. Journal of the Acoustical Society of America, 126(4): 1960-1974, October, 2009.

[7] E. C. Wilson, C. M. Reed, and L. D. Braida. Integration of auditory and vibrotactile stimuli: Effects of frequency. Journal of the Acoustical Society of America, 127(5): 3044-3059, May, 2010.

[8] E. C. Wilson, L. D. Braida and C. M. Reed Perceptual interactions in the loudness of combined auditory and vibrotactile stimuli. Journal of the Acoustical Society of America, 127(5): 3038-3043, May, 2010.

[9] C. Kayser, C. I. Petkov, M. Augath, and N. K. Logothetis. Integration of touch and sound in auditory cortex. Neuron, 48(2):373-384, October 2005.

[10] R. Okazaki, H. Kajimoto, and V. Hayward. Vibrotactile Stimulation Can Affect Auditory Loudness: A Pilot Study. Proceedings of EuroHaptics2012 (Tampere, Finland, June 12-15, 2012), Lecture Notes in Computer Science, 7283:103-108, Springer, June 2012

[11] S. J. Bolanowski, Jr., G. A. Gescheider, R. T. Verrillo, and C. M. Checkosky. Four channels mediate the mechanical aspects of touch. Journal of the Acoustical Society of America, 84(5):1680-1694, November 1988.

[12] R. Plomp and W. J. M. Levelt. Tonal Consonance and Critical Bandwidth. Journal of the Acoustical Society of America, 38(4):548-560, April 1965.

[13] C. Chafe, S. O'Modhrain. Musical muscle memory and the haptic display of performance nuance. In Proceedings of the 1996 International Computer Music Conference (Hong-Kong, China, August 1996), 1996.

[14] J. Sundberg. Chest wall vibrations in singers. Journal of Speech and Hearing Research, 26(3):329-340, September 1983. 